\title{
Concluding Summary: Theoretical Aspects
}

\author{
L. Mestel \\ Astronomy Centre, University of Sussex, \\ Falmer, Brighton BN1 9QH, England
}

To begin this formidable task I find it convenient to look back at the earliest ideas on the dynamical and thermal properties of the solar convection zone and of the mutual interaction with rotation and magnetism. The simplest picture of turbulent convection has the mixing length just an enormously increased mean-free-path, limited by the kinematic consequences of compressibility to a value of the order of the pressure scale-height. The turbulent heat transport down a superadiabatic temperature gradient is so efficient that the transport equation has to be read from right to left: Biermann and Cowling showed that in a dense convective zone the relative degree of superadiabaticity required to carry a solar luminosity is only $\cong 10^{-6}$, yielding the adiabatic $p-\rho$ relation as an excellent approximation for stellar model construction. The same mean-free-path was provisionally assumed to yield a large effective isotropic viscosity, so that the Reynolds stresses, acting alone on an arbitrary rotation field, would tend rapidly to convert it into one of uniform rotation.

A significant modification, now called the " $\Lambda$-effect", was introduced in a seminal paper by Biermann. The preferred direction of gravity builds an essential anisotropy into the turbulence, so that the Reynolds stresses no longer vanish for rigid rotation. In the original linear version, the consequent redistribution of angular momentum produces instead a zero-order radius-dependent $\Omega_{0}(r)$. This however is inconsistent with the hydrostatic equilibrium of a nearly adiabatic domain, which requires that $\Omega$ be more nearly a function of the axial distance $\varpi$ ("Taylor columns"). The consequent imbalance of meridional forces drives a laminar meridional circulation, restrained by the frictional drag due to the same anisotropic turbulence. This circulation advects angular momentum, yielding as the next approximation to the solution of the azimuthal equation of motion a latitude-dependent form for $\Omega$, which by suitable choice of parameters can be made to agree with the observed equatorial acceleration of the Sun (Kippenhahn, Köhler).

We heard from $\mathrm{Dr}$ Goode an up-to-date account of the overall solar differential rotation as inferred from helioseismology. In particular, the convection zone is now believed to rotate very similarly to the surface (with near solid body rotation beneath), without any obvious presence of Taylor columns. This is broadly consistent with the explicit ordering in the Biermann-Kippenhahn approach, which takes 
as the dominant constraint on the rotation field the azimuthal component of the equation of motion (with the anisotropic turbulence incorporated). Drs Rüdiger and Tuominen have made significant modifications, in particular by including nonlinear terms which force $\Omega$ to be a function of both $r$ and $\theta$ in advance of the effect of meridian circulation. To get good agreement with observation, however, the dominant motions affecting the angular momentum distribution must be the giant cells rather than the small-scale eddies.

Unfortunately one can study helioseismology only for the Sun. For the observed rapidly rotating late-type stars, I wonder whether the greatly increased centrifugal acceleration might not enforce a changed ordering, with the Taylor-Proudman effect dominating. A significantly differing internal rotation law would presumably modify the preferred stellar dynamo mode.

Drs Rieutord and Zahn question the validity of parametrizing the mean stress generated by the small-scale motions as an effective anisotropic viscosity (a complementary argument to the Rüdiger-Tuominen emphasis on the role of giant cells rather than small-scale eddies). However, they also state that their porous medium model does seem to give results similar to those resulting from anisotropic viscosity.

Several papers reported on new "first-principles", linear stability analysis. Kwing Chan and H.G. Mayr have introduced density stratification into the model of differential rotation as the zonal wind present in a heliostrophic eigenmode. Paul Roberts and colleagues have responded to the challenge set by Elisabeth Ribes's observations which suggest that near the solar surface the postulated solar giant cells form a "toroidal" or "doughnut" pattern rather than the predicted "cartridge-belt" or "banana-cell" pattern. They include in their zero-order state a (dynamo-generated), primarily toroidal magnetic field $\boldsymbol{B}$. The crucial parameter is the Elsasser number $\sigma B^{2} / 2 \Omega \rho$ with $\sigma$ the (turbulent) conductivity. In the low-density surface regions the Lorentz force dominates over the Coriolis force, and convective cells do indeed align themselves about the toroidal $\boldsymbol{B}$. Such linear analysis gives valuable insight and is complementary to the phenomenological attempts to model developed turbulence. I recall earlier work by Busse which can be interpreted as showing how unstable modes in a rotating fluid with (isotropic) micro-viscosity leads to an angular momentum transport which can be mimicked by an anisotropic macro-viscosity.

I was particularly intrigued by Vittorio Canuto's critique of standard mixing length theory, because of its use of a one-eddy model (appropriate for systems with high rather than low micro-viscosity) and for its postulating rather than calculating the mixing length. His prescription for a more sophisticated model includes a whole spectrum of turbulent eddies, with compressibility effects represented by taking the mixing length to be equal to the local depth. He is able to predict very closely the solar effective temperature, without any free parameters, and finds a significant reduction in the age of a globular cluster as is found from the mass at the giant branch turn-off. This work may therefore have reverberations in the ongoing debate about the value of Hubble's constant.

Dynamo theory grew out of the need to evade the consequences of Cowling's theorem, which illustrates (in Elsasser's words) the topological asymmetry between 
poloidal fields $\boldsymbol{B}_{\mathrm{p}}$ and toroidal fields $\boldsymbol{B}_{\mathrm{t}}$. In axial symmetry, it is trivially easy to generate $\boldsymbol{B}_{\mathfrak{t}}$ from $\boldsymbol{B}_{\mathrm{p}}$ via non-uniform rotation, whereas strictly axisymmetric motions merely advect both $\boldsymbol{B}_{\mathrm{p}}$ and $\boldsymbol{B}_{\mathrm{t}}$ but do not enable one to convert $\boldsymbol{B}_{\mathrm{t}}$ into $\boldsymbol{B}_{\mathrm{p}}$. Without deviation at some level from axisymmetry, the systematic shrinkage of poloidal loops into O-type neutral points, pointed out by Cowling, cannot be offset by the generation of new poloidal flux. In a seminal paper, Gene Parker showed that non-axisymmetric motions in turbulent cells, subject to Coriolis force, twist $\boldsymbol{B}_{\mathrm{t}}$ so as to yield new $\boldsymbol{B}_{\mathrm{p}}$ lines, so that in a stratified medium there is a net generation of poloidal flux. The process can be parametrized as yielding an emf along $\boldsymbol{B}$. The theory was later developed independently by Steenbeck, Krause and Rädler with the crucial new term in Ohm's law written as $\alpha B$, whence the " $\alpha$ effect" nomenclature. A whole industry has grown up studying in particular the solar dynamo, the solar cycle and its "butterfly diagram", dynamos in other solartype stars, in early-type stars with convective cores, in planets and in galaxies and galactic clusters.

We heard many variations on this theme, showing that the richness of the mhd equations with the $\alpha$-effect included is nowhere near being fully explored. Of the poster papers I liked particularly those on the " $\alpha \Lambda$-dynamos" of the HelsinkiManchester-Toulouse-Potsdam collaboration, which use in the kinematic dynamo equation a differential rotation and meridian circulation derived from the $\Lambda$-effect. (However, I would like the authors to iron out any differences among themselves - should the Sun have Taylor columns or not?). As emphasized in several papers, estimates of the field amplitudes and of filling factors must be.through effective deviation from linear kinematic theory, eg by non-linear quenching of the $\alpha$-effect (David Moss, Karl-Heinz Rädler), or by flux loss by buoyancy (Durney and Robinson, Montesinos et al.).

So much is coming out of the "standard" dynamo equations that it is salutary to be reminded by one of the founding fathers - Fritz Krause - of the probably over-simple parametrization employed, eg in the adoption of $\alpha \propto$ local helicity. The advent of new computers may enable one to avoid such parametrization by direct solution of the basic mhd equations in low-symmetry geometry. Once solutions with growing large-scale fields have been found, then one can perform the same averaging over the solutions as is at present done to the equations before their solution is attempted, if only to test the reliability of the standard procedure. I am however glad that $\mathrm{Dr}$ Chan, with his wide experience of the opportunities afforded by computers, went out of his way to emphasize the pitfalls of numerical work. In another area - interstellar magnetogasdynamics and star formation - the effect of numerical viscosity (whether explicit or implicit) is often apparent, when eg numerical solutions of the infinitely conducting mhd equations show spurious deviations from flux freezing. One might imagine that in problems with strong phenomenological turbulent viscosity or resistivity, such consequences of numerical approximation would be ignorable. But $\mathrm{Dr}$ Chan noted other dangers: for example, numerical simulation of solar convection can exaggerate the approximation to adiabaticity and so lead to a spurious prediction of Taylor columns. The 
lesson is, as always, that the computer is no substitute for the brain, and that it must be kept as a slave, not allowed to become a master.

Paul Roberts's discussion of both the similarities and the differences between the geo- and solar dynamos was particularly enlightening. Another high spot of the meeting for me was the review by Axel Brandenburg and Ilkka Tuominen of the current state of the solar dynamo. Again recalling Fritz Krause's caveats, it is salutary to have some correction to the euphoria that periodically overtakes us. Their paper stressed less the considerable achievements than the persisting problems - "there is as yet no good solar dynamo". Of special interest is the buoyancy question which has been for so long at the centre of discussion, focusing attention on the interface between the radiative core and the convective envelope. I note that Axel and Ilkka argue that loss by buoyancy is not in fact a real problem - there is rather a tendency for downward suction, so that the case for dynamo action in a boundary layer instead of in the bulk of the convective core is weakened. This is contrary both to the earlier general opinion and to the claim by $\mathrm{Dr}$ Petrovay in his paper that topological pumping will strengthen rather than weaken the buoyancy. Resolution of this disagreement on a fundamental issue is urgent. Dr Petrovay infers from his analysis a much deeper effective convection zone for the Sun. Some increase over the standard depth could be convenient for other questions, such as Li-burning, but as emphasized by Dr Goode, helioseismology sets severe limitations.

Extrapolation of solar-type dynamos to stars with higher rotation rates is encouraged by the apparent correlation between chromospheric emission and the dynamo number $\propto\left(\Omega \tau_{\mathrm{c}}\right)^{2} \propto$ (Rossby number $)^{2}$, where $\tau_{\mathrm{c}}$ is the convective turnover time at the base of the convective zone (Noyes, Vaughan et al.). Elsewhere Noyes, Weiss and Vaughan argue for a "solar cycle" period $\propto 1 / \Omega$ in those solar-type stars which do show quasi-periodic activity. However Marcello Rodono at Troms $\varnothing$ in 1987, and some participants here have queried whether the observations do point clearly to Rossby number dependence rather than just correlation with $\Omega$. I would like to see a concensus from the observers on the extent to which these relations are good guides for the theorist. Note also the warning from Dr Zeilik and others against using the solar dynamo as a universal paradigm; in particular, RS CVn activity is not well described as of solar type.

Dynamo theorists have tended to wait anxiously for information about the $\Omega$ gradient within the solar convection zone, for the sign of $\alpha \partial \Omega / \partial r$ determines the direction of dynamo wave propagation. I note the paper by Gaetano Belvedere and colleagues which exploits the helioseismological inference of a latitude dependence in $\partial \Omega / \partial r$. Nevertheless, I continue to wonder whether interpretation of the sunspot zone migration as a pure dynamo wave phenomenon is absolutely compelling. A laminar circulation of quite modest speed would easily offset a "wrong" sense of wave propagation - in Gene Parker's words at Heidelberg (1989), one could walk at the rate required without any difficulty. This underlines the importance of having a reliable theory not only of the rotation law but of the dynamically linked meridian circulation field. 
I won't disguise my (hopefully not premature) relief on hearing from Dr Goode that the 1984 hint of a rapidly rotating solar core appears now as an artefact of the method of data inversion, for such a feature if long lived sets severe if not implausible limits on the strength of any relic $\boldsymbol{B}$-field. To date it appears that we have no reliable information about the rotation of regions with $r<0.3 R$.

The organizers rightly decided not to allocate time to the detailed dynamics of magnetic braking, as there was a whole meeting later in the summer at Noto, Sicily devoted to the Angular Momentum of Young Stars. Nevertheless there was inevitably some discussion of observations and the quotation of simple theoretical formulae. Evidence for dynamo-generated magnetic control of stellar rotation was well brought out by David Gray in his paper on G-giants. The basic theoretical result on braking by a magnetically coupled wind is that of effective corotation: the rate of transport of angular momentum carried jointly by the magnetic and material stresses is equivalent to what would be carried by the gas alone if it were kept in strict corotation with the star out to the surface $S_{\mathrm{A}}$ where the wind speed reaches the local Alfven speed. The simplest form of the theory assumes just thermal driving of the wind, strong core-envelope coupling, no significant dependence of the wind temperature on rotation, a linear dynamo law relating the surface field $B_{\mathrm{s}}$ with $\Omega$, and the whole corona's participation in the wind. The consequent braking rate $\dot{\Omega} \propto-\Omega^{3}$ yields the relation $\Omega \propto t^{-\frac{1}{2}}$, prima facie consistent with observations of line widths in the solar-type stars in the Hyades, Pleiades and $\alpha$ Persei clusters. For very young stars, however, this relation appears inappropriate: David Gray and more recently Stauffer and Hartmann have produced evidence for a rapid early spin-down on the main sequence, after which something like the Skumanich $t^{-\frac{1}{2}}$ law takes over. The various corrections to the simple model suggested by theory and observation - coronal dead zones, centrifugal wind driving, a possible increase of wind temperature with $B_{\mathbf{s}}$ and hence with $\Omega$ - all act to reduce the braking rate somewhat.

During these discussions reference was made to the Stauffer-Hartmann suggestion that the apparent rapid initial spin-down is just of the convective envelope, which has only $\cong 1 / 20$ of the moment of inertia of the whole Sun. But as noted by Ian Roxburgh, a star of the age of the Sun still has had to get rid of most of its angular momentum (recall that there is at the moment no clear evidence of even a moderate differential rotation between the solar core and its convective envelope). The picture would require the transfer time of angular momentum from the core to the envelope to be longer than the time for the initial rapid braking of the envelope, which therefore spins down until the loss of angular momentum from the surface falls to a value comparable with the supply from the core. Calculations made after the meeting by my student $\mathrm{Li}$ Jianke suggest that such a model may indeed be consistent with observation, provided effective core-envelope coupling is re-established soon after the early rapid braking phase, and careful account is taken of the actual distributions of rotation rates among stars of a given type in clusters of different ages.

The rapidly accumulating wealth of observational data will require for detailed interpretation the construction of the coronal magnetic, density, temperature and 
velocity fields in terms of the dynamo-built magnetic flux distribution over the photosphere. It is by no means obvious that a simple dipolar field is appropriate for all rotation rates up to the maximum ( $c f$. the paper by Dr Katsova), or that $B_{\mathrm{s}}$ continues to scale with $\Omega$. One can however expect all fields to have a multiple structure, with closed field-line "dead zones", of high temperature and so bright in $\mathrm{X}$-rays, immersed in the cooler open-field wind regions observed as coronal holes. Recent observations of particular interest are of the rapidly rotating dwarf star AB Doradus by Cameron and Robinson, referred to in the paper by $\mathrm{Dr}$ Stewart and colleagues, and by $\mathrm{Dr}$ Vilhu during the discussions. X-ray observations reveal an extensive, very hot zone $\left(T>10^{7} \mathrm{~K}\right)$ containing cool condensations picked up in $\mathrm{H} \alpha$ absorption, corotating with the star, and showing also evidence of slow outward motion. The results can be understood semi-quantitatively in terms of compression of gas within a "dead zone" but beyond the centrifugal-gravitational balance point, and of consequent radiative cooling. The "dead zones" are thus not fully dead, but contribute to magnetic braking through the bursting out of these corotating condensations. However, I think it will be difficult to predict a total angular momentum loss greater than that given by a Weber-Davis type field, without any dead zones and, so with the whole corona taking part in the wind. The efficiency of braking depends most critically on the strength of the surface field, which fixes the field strength at the Alfvénic surface. Detailed comparison with observations of rapidly rotating young stars could supply a hint on the $B_{\mathbf{s}}(\Omega)$ dynamo relation at high rotation.

Most of the discussion has been of magnetic fields generated and maintained by contemporary dynamo action in convective zones. These zones are by definition spontaneously unstable to the continuous conversion of thermal energy into turbulent energy, and interaction of the turbulent cells with the rotation yields the degree of anisotropy capable of generating a large-scale dipolar field. There has been some limited discussion also of "fossil" magnetic fields, slowly decaying relics either of the galactic field threading the gas from which the stars formed, or of a field built up by dynamo action in an earlier epoch of the star's life. A weak fossil field threading a turbulent zone will at least initially be passive: the back-reaction of the field on the turbulence is unimportant, and the compression of the field into filaments and the possible expulsion of the field from the zone can be described in terms of the same "turbulent resistivity" as appears in the dynamo equations. A sub-adiabatic zone (without a large rotational shear) is stable against spontaneous convection: perturbing motions with a radial component are prevented from growing by the adiabatic stabilization term in the energy principle. But the presence in a sub-adiabatic zone of a magnetic field can lead to new instabilities: for motions that are non-axisymmetric but essentially horizontal, the adiabatic term vanishes, and fields of simple topology - eg purely poloidal or toroidal fields, with O-type neutral points - will spontaneously convert magnetic into kinetic energy. There is no nonlinear theory extant but it is at least plausible that a necessary condition for dynamical stability is that the field be of complex topology, with poloidal flux linking toroidal loop and vice versa. Fields that have been built up by standard dynamo action will automatically have acquired this topology. 
We were reminded by John Landstreet of the other broad class of magnetic stars - the early type magnetic Ap stars with sub-photospheric radiative envelopes. They have large-scale, well-ordered fields, apparently quasi-steady in the frames rotating with the stars, and with much higher mean strengths than the fields in the late-type main sequence stars. They can clearly take up a Conference on their own; even the debate about the origin and nature of the fields - as fossils or as the result of contemporary dynamo action in the stellar convective cores - is unresolved. $\mathrm{Dr}$ Landstreet drew attention to problems that may be awaiting us in the boundary area between the Ap and the solar-type stars. The rapid decrease at type $F$ in the extent of the outer convection zone with increasing $T_{\mathrm{e}}$ is consistent with the disappearance of the solar-type phenomenon, but it is not obvious how to explain the apparent absence of the Ap phenomenon among mid-F stars. We should remain aware of this contiguous area of magnetic star research, noting also that fossil fields may be significant in the late-type main sequence and pre-main sequence stars, eg (as already noted) by coupling the rotations of core and envelope. Dr Dudorov argues that fossil flux in fact plays an important role in the observable layers of the Sun and other solar-type stars.

I found particularly exciting the report by Drs Basri and Marcy of a strong field in a T Tauri star. It is a difficult but important task to try and decide whether all this flux is dynamo-built, or whether (as suggested by Roger Tayler) there is evidence from the activity/Rossby number curve for pre-main sequence stars of a superposition of dynamo-built and fossil fields. An Alfvén-wave driven wind is the essential feature of the T Tauri model of Teresa Lago, Michael Penston and others. And there remains the possibility (first suggested I think by Fred Hoyle) that the energy source in the T Tauri phenomenon is the dissipation of galactic magnetic flux trapped in the star during its formation.

There are many other papers in the published proceedings which will repay careful study. Those by Ed Spiegel on waves in the solar cycle, and Sasha Ruzmaikin and colleagues on the fractal distribution of solar fields impressed me particularly as being forward-looking. Our collective thanks to Ilkka Tuominen and colleagues for organizing an enjoyable and scientifically fruitful meeting. 